\title{
COHOMOLOGY AND WEIGHT SYSTEMS FOR NILPOTENT LIE ALGEBRAS
}

BY G. LEGER AND E. LUKS

Communicated by Dock Rim, July 17, 1973

1. This paper announces results concerning the cohomology groups $H^{*}(N, N)^{T}$ where $N$ is in a certain class of finite-dimensional nilpotent Lie algebras over a field $k$ and $T$ is an abelian Lie algebra faithfully represented as a maximal diagonalizable algebra of derivations of $N$; we shall refer to such an $N$ as a $T$-algebra. The additional hypotheses to be placed on the pair $N, T$ are inspired by the case when $T$ is a Cartan subalgebra and $T+N=B$ is a Borel subalgebra of a complex semisimple Lie algebra. In that case Kostant has shown [2] that $H^{i}(N, N)^{T}=0$ for $i \geqq 2$ and the authors applied this result in [3] to conclude that $H^{*}(B, B)=$ 0 . (A similar argument shows $H^{*}(P, P)=0$ for $P$ parabolic.) Here we are concerned with the relations between the vanishing of $H^{i}(N, N)^{T}$, especially for $i=2$, and the structure of the algebras $N$.

Let $W$ denote the set of weights of $T$ in $N$. If $\operatorname{dim}(T)=\operatorname{dim}\left(N / N^{2}\right)=m$ then the subset of $W$ arising from the induced representation of $T$ on $N / N^{2}$ has precisely $m$ elements, say $\left\{\alpha_{1}, \cdots, \alpha_{m}\right\}$. Every $\alpha \in W$ then has a unique representation $\alpha=\sum c_{i} \alpha_{i}$ with each $c_{i}$ a nonnegative integer and $c_{i}<p$ if the characteristic of $k$ is $p>0$. For such an $\alpha$ we call the sum (in $Z$ ) $\sum c_{i}$ the height of $\alpha$ and denote it by $|\alpha|$. For $\alpha$ in $W$, denote by $N_{\alpha}$ the weight space for $\alpha$ in $N$.

DEFINITION. A $T$-algebra is called positive if

(i) $\operatorname{dim}(T)=\operatorname{dim}\left(N / N^{2}\right)$,

(ii) $N$ is graded by the heights of the weights, i.e., if $N(j)=\bigoplus_{|a|=j} N_{\alpha}$ then $[N(j), N(k)] \subset N(j+k)$.

REMARK. Condition (ii) is superfluous in characteristic 0. However, in characteristic $p>0$ it has such consequences as $\hat{N}^{r}=0$ for $r>(p-1) \cdot \operatorname{dim}(T)$.

2. When $T$ is a Cartan subalgebra of a complex semisimple Lie algebra $G, T+N$ a Borel subalgebra of $G$ and $W$ the weights of $T$ in $N$, it is well known that $N$ is the unique positive $T$-algebra with corresponding weight system $W$. This fact is a special case of the following theorem.

AMS (MOS) subject classifications (1970). Primary 18H25, 17B30; Secondary 05 C99.

Key words and phrases. Lie algebra cohomology, weight systems, deformations of Lie algebras. 
THEOREM 1. Let $N$ be a positive T-algebra and suppose that $H^{2}\left(N / N^{r}, N / N^{r}\right)^{T}=0$ for $r \geqq 4$. If $N^{\prime}$ is any other positive T-algebra inducing the same weight system $W$ with the same height function and $\operatorname{dim}\left(N_{\alpha}^{\prime}\right) \geqq \operatorname{dim}\left(N_{\alpha}\right)$ for each $\alpha \in W$, then $N^{\prime}$ is necessarily $T$-isomorphic to $N$.

Although the task of verifying the $H^{2}$ hypothesis in Theorem 1 appears to be quite formidable, we are in fact able to check this condition for a large class of algebras by means of simple observations about $W$. For example, the collection of algebras described in [3, §5], which include the maximal nilpotent ideals of Borel subalgebras, satisfies the assumption. Also, we can show that the collection includes all the positive $T$-algebras $N$ in characteristic $\neq 2$ which satisfy the following:

If $\alpha_{1}, \cdots, \alpha_{m}$ are the elements in $W$ of height 1 , then

(i) Every element in $W$ is of the form $\alpha_{i_{1}}+\alpha_{i_{2}}+\cdots+\alpha_{i_{s}}$

(*) with $1 \leqq i_{1}<i_{2}<\cdots<i_{s} \leqq m$.

(ii) If $\gamma, \gamma-\alpha_{i}, \gamma-\alpha_{j} \in W$ with $i \neq j,|\gamma| \geqq 3$ then $\gamma-\alpha_{i}-\alpha_{j} \in W$ and $\alpha_{i}+\alpha_{j} \notin W$.

3. The appearance of $H^{2}\left(N / N^{r}, N / N^{r}\right)^{T}$ in Theorem 1 is suggestive of the role that $H^{2}(N, N)^{T}$ plays in a rigidity theorem for algebras acted upon by $T$. The set of $T$ multiplications in a vector space $N$ on which $T$ operates clearly form an algebraic subvariety of the affine space $N^{*} \wedge N^{*} \otimes N$. Thus we are led to a restricted deformation theory for such algebras. If $T$ operates diagonally on $N$ then $H^{*}(N, N)^{T}$ plays the role in this theory that $H^{*}(L, L)$ plays in the deformation theory of ordinary Lie algebras (cf. [4]). For example, we have the concept of $T$-rigidity and the theorem: If $\mu$ is a $T$-multiplication on $N$ and if $H^{2}((N, \mu),(N, \mu))^{T}=0$ then $(N, \mu)$ is $T$-rigid. Also, $H^{3}((N, \mu),(N, \mu))^{T}$ arises as obstructions to integrability of 2-cocycles.

We remark that the hypothesis $H^{2}\left(N / N^{r}, N / N^{r}\right)^{T}=0$, for $r \geqq 4$, of Theorem 1 is strictly stronger for positive $T$-algebras than the vanishing of $H^{2}(N, N)^{T}$.

4. Suppose characteristic $(k) \neq 2$. A remarkable class of positive $T$-algebras over $k$ is obtained by generalizing the Coxeter-Dynkin diagram for $A_{l}$.

Let $\Gamma$ be an undirected graph [5] with at most one edge connecting any two vertices and without loops (i.e., for any vertex $v,(v, v)$ is not an edge). A section graph $g$ of $\Gamma$ is a subgraph such that any edge in $\Gamma$ which connects two vertices of $g$ is in $g$. By a subtree $t$ of $\Gamma$ we shall mean a connected section graph with no circuits.

Definition. A collection $S$ of subtrees of $\Gamma$ will be called admissible if

(i) whenever $t \in S$ and $t^{\prime}$ is a subtree of $t$ then $t^{\prime} \in S$. 
(ii) $\Gamma=\bigcup_{t \in S} t$. (So each vertex and each edge in $\Gamma$ is in some $t$ of the collection.)

To each admissible collection $S$ of subtrees of $\Gamma$ we associate a Lie algebra $N_{S}$ as follows:

Arbitrarily assign a direction $v_{i} \rightarrow v_{j}$ to each edge $\left(v_{i}, v_{j}\right)$ in $\Gamma$. We define $\varepsilon: S \times S \rightarrow\{ \pm 1,0\}$ such that $\varepsilon_{t, t^{\prime}}=0$ if either $t \cap t^{\prime} \neq \varnothing$ or $t \cup t^{\prime} \notin S$, otherwise $\varepsilon_{t, t^{\prime}}=1$ if the unique edge connecting $t$ to $t^{\prime}$ is directed $t \rightarrow t^{\prime}$ and $\varepsilon_{t, t^{\prime}}=-1$ if this edge is directed $t^{\prime} \rightarrow t$. Now let $N_{S}$ be the $k$-algebra with basis $S$ such that $\left[t, t^{\prime}\right]=\varepsilon_{t, t} t \cup t^{\prime}$.

The Jacobi identity is readily verified and so $N_{S}$ is a nilpotent Lie algebra generated by the set of vertices. Next we point out that, for any vertex $v$ of $\Gamma$, there is a unique derivation $a_{v}$ of $N_{S}$ such that $a_{v}(v)=v$ and $a_{v}(w)=0$ for any vertex $w \neq v$. The set of derivations $a_{v}$ span a maximal diagonizable algebra $T_{S}$ of derivations of $N_{S}$. One sees that $N_{S}$ is a positive $T_{S^{-}}$-algebra and it is easy to verify that it satisfies property $(*)$. Thus, by Theorem $1, N_{S}$ is the unique $T_{S}$-algebra producing its system of weights. In particular, its isomorphism class is independent of the choice of directions in $\Gamma$.

The simplest of graphs consists of a single path and if, in this case, $S$ is taken to be the collection of all subtrees, $N_{S}$ is the nilpotent subalgebra corresponding to the positive weights in an algebra of type $A_{l}$.

Thus, it is not surprising that the algebras $N_{S}$ have structural and cohomological properties like those of the maximal nilpotent subalgebras of Borel subalgebras. For example, using the classical spectral sequences of Hochschild-Serre [1] as well as combinatorial properties of graphs, we can show:

THEOREM 2. Let characteristic $(k)=0$. Suppose $\Gamma$ is a graph, $S$ an admissible collection of subtrees. Then $H^{i}\left(N_{S}, N_{S}\right)^{T_{S}}=0$ for $i \geqq 2$.

Then, following methods used in [3], we prove:

THEOREM 3. Let characteristic $(k)=0$. If $B_{S}$ is the semidirect sum $T_{S}+N_{S}$ then $H^{*}\left(B_{S}, B_{S}\right)=0$.

Finally, we announce a characterization of the graph algebras by property (*). First we assign to each positive $T$-algebra $N$ a graph $\Gamma(N)$ as follows: Let $\alpha_{1}, \cdots, \alpha_{m}$ be the elements in $W$ of height 1 , then $\Gamma(N)$ is the graph on $m$ vertices $v_{1}, \cdots, v_{m}$ such that $\left(v_{i}, v_{j}\right)$ is an edge if and only if $\alpha_{i}+\alpha_{j}$ is in $W$. Let $S$ be any admissible collection of subtrees of $\Gamma(N)$. Then $T$ acts on $N_{S}$ via the isomorphism $T \rightarrow T_{S}$ given by $x \rightarrow$ $\sum_{i} \alpha_{i}(x) a_{v_{i}}$. 
THEOREM 4. Let $N$ be a positive T-algebra satisfying property (*). For each $\alpha=\alpha_{i_{1}}+\cdots+\alpha_{i_{s}}$ in $W$, let $g_{\alpha}$ be the section graph of $\Gamma(N)$ with vertices $v_{i_{1}}, \cdots, v_{i_{s}}$. Then each such $g_{\alpha}$ is a subtree of $\Gamma(N)$; the set $S=\left\{g_{\alpha} \mid \alpha \in W\right\}$ is an admissible collection of subtrees of $\Gamma(N)$; and $N$ is T-isomorphic to $N_{S}$.

\section{REFERENCES}

1. G. Hochschild and J.-P. Serre, Cohomology of Lie algebras, Ann. of Math. (2) 57 (1953), 591-603. MR 14, 943.

2. B. Kostant, Lie algebra cohomology and generalized Schubert cells, Ann. of Math. (2) 77 (1963), 72-144. MR 26 \#266.

3. G. Leger and E. Luks, Cohomology theorems for Borel-like solvable Lie algebras in arbitrary characteristic, Canad. J. Math. 24 (1972), 1019-1026.

4. A. Nijenhuis and R. Richardson, Cohomology and deformations in graded Lie algebras, Bull. Amer. Math. Soc. 72 (1966), 1-29. MR 33 \#4190.

5. O. Ore, Theory of graphs, Amer. Math. Soc. Colloq. Publ., vol. 38, Amer. Math. Soc. Providence, R. I., 1962. MR 27 \#740.

\footnotetext{
Department of Mathematics, Tufts University, Medford, Massachusetts 02155
}

Department of Mathematics, Bucknell University, Lewisburg, Pennsylvania 17837 